\title{
Condiciones para el aprendizaje organizacional y prácticas de gestión de innovación: un análisis en medianas empresas
}

\author{
Vanessa Pertuz* y Adith Pérez \\ Facultad de Ingenierías y Tecnológicas, Universidad Popular del Cesar, Valledupar, Colombia. \\ (correo-e: adithperez@unicesar.edu.co; vanessapertuz@unicesar.edu.co) \\ * Autor a quien debe ser dirigida la correspondencia
}

Recibido Dic. 17, 2019; Aceptado Feb. 14, 2020; Versión final Mar. 10, 2020, Publicado Jun. 2020

\begin{abstract}
Resumen
El objetivo de este artículo es describir las condiciones de aprendizaje organizacional y las prácticas que fomentan la innovación en las medianas empresas. La investigación se fundamenta epistemológicamente en el positivismo lógico, con un enfoque cuantitativo de tipo descriptivo. La muestra corresponde a medianas empresas del municipio de Valledupar, Colombia. La investigación utiliza la encuesta como técnica de recolección de datos y un cuestionario con escala tipo Likert. La validez de contenido del cuestionario se establece mediante juicio de expertos. La confiabilidad se determina utilizando el coeficiente de Alfa de Cronbach. Los resultados indican que las empresas analizadas fomentan los procesos de aprendizaje mediante la cultura organizacional, la formación y la articulación de la estrategia competitiva. Se concluye que las medianas empresas implementan la generación y selección de ideas como práctica de gestión de innovación. Sin embargo, presentan dificultades en la gestión de recursos para el desarrollo de proyectos innovadores.
\end{abstract}

Palabras clave: condiciones de aprendizaje organizacional; prácticas de gestión de innovación; medianas empresas

\section{Organizational learning conditions and innovation management practices: an analysis of medium-sized firms}

\begin{abstract}
This article aims to describe the organizational learning conditions and practices that promote innovation in medium-sized companies. The research is based on logical positivism using a descriptive and quantitative approach. The sample is composed of medium-sized companies from the municipality of Valledupar, Colombia. A survey and a questionnaire with a Likert-type scale are employed for data collection. The questionnaire's content validity is estimated by expert judgment. The reliability is determined using the Cronbach's alpha coefficient. The results indicate that the companies analyzed promote learning processes through organizational culture, training, and the articulation of competitive strategy. In conclusion, companies implement the generation and selection of ideas as an innovation management practice. However, they present difficulties in managing resources for the development of innovative projects.
\end{abstract}

Keywords: organizational learning conditions; innovation management practices; medium-sized firms 


\section{INTRODUCCIÓN}

En la actualidad, el aprendizaje organizacional corresponde a un elemento fundamental para las organizaciones, este proceso favorece la generación de ideas, la identificación de nuevas oportunidades de mercado, y consecuentemente, el desarrollo de innovaciones. De esta manera, es relevante analizar las condiciones de la organización que posibilitan el aprendizaje entre sus miembros. Al respecto, Stable (2016), señala que no existe consenso en la literatura frente a las condiciones de aprendizaje organizacional, sin embargo, estas pueden maximizarse cuando se presentan las condiciones organizacionales adecuadas (Castañeda, 2015). En la revisión de literatura se destacan diferentes condiciones que favorecen el aprendizaje organizacional, tales como: la cultura de aprendizaje organizacional, la formación, la claridad estratégica y el soporte organizacional (Castañeda, 2015).

\section{CONDICIONES PARA EL APRENDIZAJE ORGANIZACIONAL}

A continuación, se presentan las definiciones de las condiciones para el aprendizaje organizacional analizadas: cultura de aprendizaje, formación, claridad estratégica y soporte organizacional.

\section{Cultura de aprendizaje}

La cultura es un elemento ligado al aprendizaje y la transformación de las organizaciones, mediante dos niveles fundamentales: los rasgos de las personas (aprendizaje continuo, indagación, dialogo y empoderamiento) y la estructura de la organización (sistemas de gestión y liderazgo estratégico) (Xie et al., 2019). Así, el concepto de cultura de aprendizaje (desde los constructos de organización de aprendizaje y cultura organizacional) se define como un conjunto de valores y actitudes hacia el aprendizaje (Gil y Mataveli, 2017). Este tipo de cultura se caracteriza por la relación entre el intercambio de conocimientos y la solución de problemas (Castañeda, 2015) y por modificar sus comportamientos a partir de la capacidad de crear, adquirir y transferir conocimiento (Xie et al., 2019).

En este sentido, la cultura de aprendizaje aumenta el rendimiento (Xie et al., 2019), la adaptación al cambio (Malik y Garg, 2017), la ventaja competitiva sostenible (Overstreet et al., 2019), el empoderamiento en las competencias y el trabajo en equipo (Gupta et al., 2019). Asimismo, la cultura de aprendizaje fomenta la innovación, la calidad, la competitividad y la promoción del desarrollo de los colaboradores (Cavazotte et al., 2015). Específicamente en el contexto de las pequeñas y medianas empresas (PYMES), la cultura de aprendizaje es una condición fundamental para el éxito y el desarrollo (Xie et al., 2019).

\section{Formación}

La formación es un elemento importante en las culturas organizacionales de alto desempeño (Ibrahim et al,, 2017; Berber y Lekovic, 2018; Xiao et al., 2019) y en la consolidación de ventajas competitivas (Padachi y Lukea, 2016; Manresa et al., 2019) debido a que contribuye al empoderamiento (Natalicchio et al., 2018), las oportunidades de desarrollo de los colaboradores (Xiao et al., 2019) y su autoeficacia, mediante la adquisición del conocimiento clave para impactar los objetivos de la organización (Castañeda, 2015).

De esta manera, los procesos de formación son relevantes en el contexto organizacional para promover la efectividad de la innovación (Berber y Lekovic, 2018; Roshchin y Travkin, 2017; Manresa et al., 2019). De allí que, en la actualidad, se fomenta la capacitación de los colaboradores en habilidades duras y blandas (Ibrahim et al., 2017). Igualmente, en el contexto de las PYMES es importante favorecer el aprendizaje a través de diferentes mecanismos de formación (Yap y Toh, 2020). No obstante, este tipo de empresas, frecuentemente, carecen de los recursos necesarios para emprender procesos de formación y enfrentan barreras en materia de la percepción de los gerentes, los costos, el financiamiento, las presiones externas, el ciclo de capacitación y el diseño de capacitación (Padachi y Lukea, 2016). De igual forma, el estudio de Anlesinya (2018) identifica cuatro barreras comunes para la capacitación: la falta de una cultura participativa en la toma de decisiones, el escaso compromiso de la alta dirección, la falta de promoción y crecimiento profesional que ofrece la empresa y finalmente, la falta de transparencia y equidad en la selección de los beneficiarios de los procesos de formación.

\section{Claridad estratégica}

El éxito de las iniciativas basadas en conocimiento depende de su alineación con la estrategia de la organización, en este contexto, la claridad estratégica se concibe como el conocimiento de los colaboradores respecto a la planeación organizacional (Castañeda, 2015). Así, el desafío de la dirección estratégica es posicionar la innovación en la empresa, mediante el trabajo colaborativo tanto interno como externo, que permita aumentar la competitividad (Álvarez y Bernal, 2017) y consolidar ecosistemas de 
innovación (Dias et al., 2020). Además, el conocimiento de la estrategia aumenta la motivación de los colaboradores debido a que identifican su aporte al logro de los objetivos (Castañeda, 2015) y es un factor determinante de la relación entre la estrategia y el desempeño, en estructuras descentralizadas (Qinglan et al., 2019).

\section{Soporte organizacional}

El soporte organizacional se define como la disponibilidad de recursos físicos y tecnológicos de la organización para compartir conocimiento y promover el aprendizaje (Castañeda, 2015). Al respecto, el apoyo organizacional tiene un efecto positivo en el desempeño de los colaboradores, la innovación (Suifan et al., 2018; Chen et al., 2020) y la capacidad de respuesta a los cambios del entorno (Ferreira et al., 2018). Además, el apoyo organizacional percibido aumenta la creatividad y el sentido de pertenencia (Suifan et al., 2018; Tang et al., 2017), al tiempo que mejora el ambiente de trabajo, el compromiso (Arasanmi y Krishna, 2019) y la integración organizacional (Singh, 2019). De acuerdo con los planteamientos anteriores, la Tabla 1 presenta los aspectos observados respecto a las condiciones de aprendizaje organizacional.

Tabla 1: Aspectos observados en las condiciones de aprendizaje organizacional

\begin{tabular}{|l|l|}
\hline $\begin{array}{c}\text { Condiciones para el } \\
\text { aprendizaje } \\
\text { organizacional }\end{array}$ & \multicolumn{1}{c|}{ Aspectos observados } \\
\hline $\begin{array}{l}\text { Cultura de } \\
\text { aprendizaje }\end{array}$ & $\begin{array}{l}\text { Los colaboradores participan activamente de la solución de los problemas. } \\
\text { Los directivos aplican el conocimiento de los colaboradores en solución de problemas. } \\
\text { Los miembros de la empresa colaboran activamente entre ellos. }\end{array}$ \\
\hline Formación & $\begin{array}{l}\text { Los directivos promueven la participación de sus colaboradores en procesos de formación. } \\
\text { Los directivos promueven el desarrollo de nuevas habilidades en los colaboradores } \\
\text { Se facilita la formación para el logro de los objetivos organizacionales. }\end{array}$ \\
\hline Claridad estratégica & $\begin{array}{l}\text { Los colaboradores conocen la misión de la empresa. } \\
\text { Los colaboradores conocen la visión de la empresa. } \\
\text { Los colaboradores conocen los objetivos estratégicos de la empresa. }\end{array}$ \\
\hline $\begin{array}{l}\text { Soporte } \\
\text { organizacional }\end{array}$ & $\begin{array}{l}\text { Los colaboradores cuentan con equipos de cómputo para facilitar el flujo de información. } \\
\text { Los colaboradores disponen de sistemas de información que faciliten el flujo de la información } \\
\text { La empresa cuenta con recursos tecnológicos para almacenar el conocimiento disponible. }\end{array}$ \\
\hline
\end{tabular}

\section{PRÁCTICAS DE GESTIÓN DE INNOVACIÓN}

De otro lado, el artículo analiza las prácticas organizacionales que favorecen la gestión de la innovación: estrategia y cultura de innovación; gestión de recursos; y generación y selección de ideas.

\section{Estrategia y cultura de innovación}

Recientemente, es el estudio de Gil et al. (2018) concluye que las condiciones con mayor impacto para facilitar la innovación son: el establecimiento de una cultura de aprendizaje y una estructura organizativa que promueva el aprendizaje. En este sentido, la comunicación, la presencia de una estrategia de innovación, la participación de los colaboradores en procesos de intercambio de conocimiento y comunidades de práctica son elementos fundamentales en la innovación empresarial (Díaz et al., 2014; González et al., 2014; Bravo y Herrera, 2009). Puntualmente, la cultura de innovación se define como la atmósfera multidimensional de valores y creencias compartidas por los equipos que los hacen propensos a explorar nuevas oportunidades y conocimientos para innovar (Naranjo y Calderon, 2018; Dabić et al., 2019; Ghasemzadeh et al., 2019). Este tipo de culturas son impulsadas por 4 aspectos fundamentales: liderazgo, recursos, gestión del conocimiento y procesos (Dobni y Klassen, 2020).

De esta manera, la teoría destaca la importancia de la orientación estratégica basada en la innovación (Norris y Ciesielska, 2019). En este sentido, el desarrollo de una cultura de innovación incluye prácticas como: el liderazgo (Villaluz y Hechanova, 2019), la interacción de las distintas áreas de la empresa para innovar (González et al., 2014; Bravo y Herrera, 2009), el fomento de la creatividad y el cambio (Díaz et al., 2014; Bravo y Herrera, 2009; Naranjo y Calderón, 2015; Tarapuez et al., 2016; Ghasemzadeh et al., 2019; Dabić et al., 2019), la interacción con la comunidad científica y los vínculos con el entorno para procesos de I+D (Bravo y Herrera, 2009; Tarapuez et al., 2016; Álvarez y Bernal, 2017) y la promoción de la participación y la autonomía de los equipos de trabajo (Bravo y Herrera, 2009; Naranjo y Calderón, 2015; Ghasemzadeh et al., 2019; Hanifah et al., 2020). Adicionalmente, los componentes del capital intelectual (humano, estructural y relacional) impactan significativamente la cultura de innovación (Dabić et al., 2019; Hanifah et al., 2020). 
Asimismo, la cultura aumenta el desempeño innovador de las organizaciones, por lo que una estrategia organizacional efectiva corresponde a desarrollar culturas basadas en una actitud abierta hacia el aprendizaje y la innovación (Hanifah et al., 2020). Al respecto, la estrategia de la innovación incluye, además del desarrollo interno de actividades de $\mathrm{I}+\mathrm{D}$, un conjunto de acciones relacionadas con la adquisición externa de conocimiento mediante de transacciones de mercado y de la cooperación con otros agentes (Vega et al., 2009). Por su parte, Clausen et al. (2011) definen la estrategia de innovación como el conjunto de decisiones de la empresa en relación con los procesos de innovación: las actividades centrales de innovación (aquellas relacionadas o no con la inversión en I+D) y las fuentes de información a utilizar externas o internas. En este sentido, una estrategia de innovación proactiva y orientada al riesgo tienen un impacto significativo en la cultura de la innovación (Díaz et al., 2014; González et al., 2014; Tarapuez et al., 2016; Hanifah et al., 2019).

\section{Gestión de los recursos}

Las empresas que invierten en innovación, logran mejor desempeño, mediante la generación de nuevos o mejorados productos y procesos (Córdoba y Naranjo, 2017). En este ámbito, los pilares fundamentales de la estrategia de innovación corresponden a los recursos, la tecnología, el marketing y la gestión (Meng y Brown, 2018). Tradicionalmente, los recursos internos de la empresa se han medido en términos de su estructura de I + D. No obstante, la literatura sugiere que diversos factores, tales como la capacitación, interna y externa, para impactar el desempeño innovador (Cainelli et al., 2015). En consecuencia, la gestión estratégica de recursos es un elemento central de la orientación estratégica para innovar (Meng y Brown, 2018)

La literatura plantea la efectividad de diferentes fuentes para gestionar recursos innovadores. En primer lugar, se destaca la importancia de obtener recursos internos para innovar (Díaz et al., 2014; Cainelli et al., 2015; Da Costa et al., 2014). En segundo lugar, algunos autores enfatizan en la explotación de los recursos existentes en la empresa (Meng y Brown, 2018), considerando que los recursos internos son los determinantes para la innovación (Abdul y Medase, 2019). En tercer lugar, se plantea la necesidad de articular los recursos internos y externos mediante procesos de comunicación y gestión del conocimiento (Díaz et al., 2014; González et al., 2014; Fernandes et al., 2018; Abdul y Medase, 2019).

\section{Generación y selección de ideas}

La revisión de literatura presenta diferentes enfoques relacionados con la generación y selección de ideas para innovar. Un primer enfoque destaca la importancia de los clientes en los procesos de ideación (González et al., 2014; Díaz et al., 2014; Cooper, 2019), mediante técnicas como la investigación etnográfica y los grupos focales que permitan identificar las necesidades del cliente (Cooper, 2019). En segundo enfoque destaca la importancia de los esfuerzos creativos de los colaboradores en la fase de generación de ideas (Bäckström y Bengtsson, 2019). El tercer enfoque corresponde a los procesos de innovación abierta (Liao et al., 2020) y los procesos de identificación de iniciativas soportados en tecnológicas. En este contexto se incluyen las plataformas de ideación basadas en la web (Qian et al., 2019; Cooper, 2019), y el análisis de redes sociales para generar innovación basada en datos (Bhimani et al., 2019). La tabla 2 presenta los aspectos teóricos observados en cada una de las prácticas de gestión de innovación analizadas en el presente artículo.

Tabla 2: Aspectos observados en las prácticas de gestión de la innovación

\begin{tabular}{|l|l|}
\hline $\begin{array}{c}\text { Práctica de gestión } \\
\text { de la innovación }\end{array}$ & \multicolumn{1}{c|}{ Aspectos observados } \\
\hline $\begin{array}{l}\text { Estrategia y cultura } \\
\text { de innovación }\end{array}$ & $\begin{array}{l}\text { La empresa comunica la estrategia de innovación a todos los colaboradores, mediante } \\
\text { diferentes canales } \\
\text { Existe una interacción permanente entre las distintas áreas para tratar temas relacionados con } \\
\text { la innovación. } \\
\text { En la empresa se favorece el ensayo y error como parte del aprendizaje para innovar en los } \\
\text { productos. }\end{array}$ \\
\hline $\begin{array}{l}\text { Gestión de los } \\
\text { recursos }\end{array}$ & $\begin{array}{l}\text { La empresa cuenta con una unidad en la que se organizan las actividades de innovación. } \\
\text { Se utilizan mecanismos de propiedad intelectual para la protección de las innovaciones. } \\
\text { La empresa cuenta con recursos para invertir en Investigación y Desarrollo. }\end{array}$ \\
\hline $\begin{array}{l}\text { Generación y } \\
\text { selección de ideas }\end{array}$ & $\begin{array}{l}\text { Se promueven por distintos medios, la participación de los colaboradores en concursos de } \\
\text { innovación. } \\
\text { Se evalúan las ideas innovadoras en función del impacto que generan en algún área de la } \\
\text { organización } \\
\text { Se priorizan las ideas innovadoras que tengan aplicabilidad inmediata para un cliente }\end{array}$ \\
\hline
\end{tabular}




\section{METODOLOGÍA}

La investigación se enmarca en la escuela de pensamiento del positivismo lógico, con un enfoque cuantitativo, de tipo descriptivo (Hernandez et al., 2014). Este trabajo considera un diseño de campo (Tamayo, 2011); no experimental y transaccional. La población está integrada por 81 medianas empresas legalmente constituidas, de acuerdo con la información reportada por la Cámara de Comercio del municipio de Valledupar, Cesar Colombia, y con domicilio en este municipio. La muestra corresponde a 44 empresas seleccionadas mediante un muestreo probabilístico, de tipo aleatorio simple (Martínez, 2012).

La investigación utiliza la encuesta como técnica de recolección de datos. El instrumento de recolección de datos corresponde a un cuestionario de preguntas cerradas, conformado por 21 ítems con escala tipo Likert con 5 opciones de respuesta: 5: Siempre, 4: Casi siempre, 3: Algunas veces, 2: Casi nunca, 1: Nunca. La validez de contenido del instrumento se estableció a partir del juicio de expertos y la confiabilidad mediante el coeficiente de Alfa de Cronbach (Hernandez et al., 2014). Para la estimación de la confiabilidad se efectuó una prueba piloto (Tamayo, 2011). Específicamente, se obtiene un coeficiente de 0,861, lo que indica que el instrumento es confiable, asegurando un alto nivel de consistencia interna en las respuestas de los informantes.

Como técnicas de análisis de datos, se utiliza la estadística descriptiva, específicamente la distribución de frecuencias, las medidas de tendencia de central y de dispersión. Para la interpretación de los datos se estableció un punto de comparación o baremo de interpretación; el cual fue construido, para efectuar el proceso de confrontación de los resultados. El baremo de la media se establece desde 1 hasta 5 debido a que corresponden al valor mínimo y máximo posible, dada la escala de respuesta del instrumento de recolección de datos. A continuación, se presenta el baremo utilizado para el análisis promedio de las variables analizadas (Tabla 3 ).

Tabla 3: Baremo ponderado para el análisis de las medias

\begin{tabular}{|c|c|c|c|c|}
\hline Valor & Alternativa & Intervalos & Categorías & Convención \\
\hline 5 & Siempre & $4,20-5,00$ & Muy presente & MP \\
\hline 4 & Casi siempre & $3,40-4,19$ & Presente & $\mathrm{P}$ \\
\hline 3 & Algunas veces & $2,60-3,39$ & Medianamente presente & MEP \\
\hline 2 & Casi nunca & $1,80-2,59$ & Poco presente & PP \\
\hline 1 & Nunca & $1,00-1,79$ & Ausente & $\mathrm{A}$ \\
\hline
\end{tabular}

Además, se calculó la desviación estándar de cada una de las variables analizadas. Para tal efecto, se establece el baremo de análisis que corresponde a un rango entre 0 y el valor máximo de la desviación estándar obtenida de la aplicación del instrumento (Tabla 4).

Tabla 4: Baremo ponderado para el análisis de la desviación estándar

\begin{tabular}{|c|c|c|c|}
\hline Valor & Intervalos & Categorías & Convención \\
\hline 5 & $1,39-1,74$ & Muy alta dispersión & MAD \\
\hline 4 & $1,05-1,38$ & Alta dispersión & ALD \\
\hline 3 & $0,70-1,04$ & Dispersión intermedia & DI \\
\hline 2 & $0,35-0,69$ & Baja dispersión & BD \\
\hline 1 & $0,00-0,34$ & Ausente dispersión & AD \\
\hline
\end{tabular}

\section{RESULTADOS}

En la Tabla 5 se presentan los resultados obtenidos frente a las condiciones para el aprendizaje organizacional en medianas empresas: cultura de aprendizaje, formación, claridad estratégica y soporte organizacional. En primera instancia, en la cultura de aprendizaje se evidencia que la mayor parte de las respuestas de los informantes se ubican en las opciones siempre $(57 \%)$ y casi siempre (20\%). En consecuencia, la cultura de aprendizaje se manifiesta como muy presente de acuerdo con el baremo establecido, con una media de 4,27. Además, se obtiene una desviación estándar de 1,00, lo que indica una dispersión intermedia de las respuestas. En este sentido, en las empresas analizadas se valida una tendencia positiva frente a la cultura de aprendizaje, basada en una relación entre el intercambio de conocimientos y la solución a los problemas de la empresa (Castañeda, 2015). Adicionalmente, la literatura destaca la importancia de los lideres para el desarrollo de una cultura de aprendizaje (Castañeda, 2015; Malik y Garg, 2017; Xie et al., 2019), mientras que Cavazotte et al. (2015) indican que los elementos característicos de la cultura permiten a las empresas valorar el aprendizaje como un elemento clave para 
sus actividades. además, estos elementos favorecen el desempeño de los colaboradores (Xie et al., 2019; Overstreet et al., 2019) y permiten gestionar los cambios del entorno (Malik y Garg, 2017; Xie et al., 2019).

Tabla 5: Resultados de las condiciones de aprendizaje organizacional

\begin{tabular}{|c|c|c|c|c|c|c|c|c|c|c|c|c|c|c|c|c|}
\hline \multirow{3}{*}{$\begin{array}{l}\text { Indicadores } \\
\text { Cultura de } \\
\text { aprendizaje }\end{array}$} & \multicolumn{2}{|c|}{ Nunca } & \multicolumn{2}{|c|}{$\begin{array}{c}\text { Casi } \\
\text { nunca }\end{array}$} & \multicolumn{2}{|c|}{$\begin{array}{c}\text { Algunas } \\
\text { veces }\end{array}$} & \multicolumn{2}{|c|}{$\begin{array}{c}\text { Casi } \\
\text { siempre }\end{array}$} & \multicolumn{2}{|c|}{ Siempre } & \multicolumn{2}{|c|}{ Total } & \multirow{2}{*}{\multicolumn{2}{|c|}{$\bar{X}$}} & \multirow{2}{*}{\multicolumn{2}{|c|}{$\sigma$}} \\
\hline & $\mathrm{Fa}$ & $\%$ & $\mathrm{Fa}$ & $\%$ & $\mathrm{Fa}$ & $\%$ & $\mathrm{Fa}$ & $\%$ & $\mathrm{Fa}$ & $\%$ & $\mathrm{Fa}$ & $\%$ & & & & \\
\hline & 1 & 2 & 1 & 2 & 8 & 18 & 9 & 20 & 25 & 57 & 44 & 100 & 4,27 & MP & 1,00 & DI \\
\hline Formación & 2 & 5 & 1 & 2 & 3 & 7 & 12 & 27 & 26 & 59 & 44 & 100 & 4,39 & MP & 0,97 & DI \\
\hline $\begin{array}{l}\text { Claridad } \\
\text { estratégica }\end{array}$ & 0 & 0 & 1 & 2 & 3 & 7 & 10 & 23 & 30 & 68 & 44 & 100 & 4,57 & MP & 0,72 & $\mathrm{DI}$ \\
\hline $\begin{array}{l}\text { Soporte } \\
\text { organizacional }\end{array}$ & 3 & 7 & 2 & 5 & 2 & 5 & 14 & 32 & 23 & 52 & 44 & 100 & 4,18 & $P$ & 1,16 & ALD \\
\hline \multicolumn{13}{|c|}{ Media $(\bar{X})$ general de la dimensión } & \multicolumn{4}{|c|}{4,35} \\
\hline \multicolumn{13}{|c|}{ Categoría general de la dimensión (Media $\bar{X}$ ) } & \multicolumn{4}{|c|}{ Muy presente (MP) } \\
\hline \multicolumn{13}{|c|}{ Desviación estándar $(\sigma)$ general de la dimensión } & \multicolumn{4}{|c|}{0,98} \\
\hline \multicolumn{13}{|c|}{ Categoría general de la dimensión (Desviación estándar $\sigma$ ) } & \multicolumn{4}{|c|}{ Dispersión Intermedia (DI) } \\
\hline
\end{tabular}

En segunda instancia, frente a la formación, el $59 \%$ de las respuestas corresponden a la opción siempre y el $27 \%$ a la opción casi siempre, validando la presencia de esta condición en las empresas analizadas, con una media de 4,39 y una desviación estándar de 0,97, lo que indica una dispersión intermedia. Estos resultados coinciden con lo planteado por Castañeda (2015), quien destaca que el aprendizaje de los miembros de la organización contribuye al logro de los objetivos laborales. Igualmente, la literatura destaca que la formación contribuye al desarrollo de competencias claves para el desempeño empresarial (Castañeda, 2015; Ibrahim et al., 2017; Berber y Lekovic, 2018; Manresa et al., 2019; Xiao et al., 2019; Yap y Toh, 2020), la ventaja competitiva y la innovación (Roshchin y Travkin, 2017; Natalicchio et al., 2018; Manresa et al., 2019).

En tercera instancia, el $68 \%$ de los informantes responde siempre y el $23 \%$ casi siempre, en los ítems relacionados con la claridad estratégica. Estos resultados indican que los colaboradores perciben claridad en la apuesta estratégica de la organización, con una media de 4,57. Asimismo, se obtiene una desviación estándar de 0,72 , indicando una dispersión intermedia. Estos resultados validan que la claridad estratégica es una condición para el aprendizaje organizacional (Castañeda, 2015). En este escenario, se destaca la importancia de la capacidad de los lideres para establecer y comunicar la estrategia de la empresa (Álvarez y Bernal, 2017), debido a que cuando los colaboradores conocen la misión, la visión y los objetivos estratégicos, es factible identificar el conocimiento clave para compartir (Castañeda, 2015) y estimular el desempeño mediante la descentralización (Qinglan y Eriksson, 2019).

En cuarta instancia, el $52 \%$ de los informantes responde siempre y el $32 \%$ casi siempre en los ítems relacionados con el soporte organizacional. Lo cual indica que las infraestructuras de soporte al conocimiento se encuentran presentes en las empresas analizadas. Al respecto se obtiene una media de 4,18 , y una desviación estándar de 1,16, lo que indica una alta dispersión de las respuestas. No obstante, esta condición obtiene la menor calificación por parte de los informantes. En este sentido, se destaca la importancia de promover la implementación de herramientas tecnológicas facilitan a los colaboradores la adquisición, creación y socialización del conocimiento (Castañeda, 2015). Adicionalmente, el apoyo organizacional percibido puede aumentar la creatividad y el compromiso de los colaboradores (Tang et al., 2017; Suifan et al., 2018; Arasanmi y Krishna, 2019; Chen et al., 2020).

En síntesis, la media ubica a las condiciones para el aprendizaje organizacional en la categoría muy presente, con un promedio de 4,35 y una desviación estándar de la dimensión de 0,98 indicando una dispersión intermedia en las respuestas. De acuerdo con lo anterior, el presente estudio valida que el aprendizaje organizacional (creación y adquisición de conocimiento) es un proceso que puede facilitarse a partir de las condiciones de las empresas (Castañeda, 2015).

De otro lado, en la Tabla 6 se presentan los resultados obtenidos de las prácticas de gestión de innovación: estrategia y cultura de innovación, gestión de los recursos, generación y selección de ideas. 
Tabla 6: Resultados de las prácticas de gestión de innovación

\begin{tabular}{|c|c|c|c|c|c|c|c|c|c|c|c|c|c|c|c|c|}
\hline \multirow{3}{*}{$\begin{array}{l}\text { Practicas } \\
\text { Estrategia y } \\
\text { cultura de } \\
\text { innovación }\end{array}$} & \multicolumn{2}{|c|}{ Nunca } & \multicolumn{2}{|c|}{$\begin{array}{c}\text { Casi } \\
\text { nunca }\end{array}$} & \multicolumn{2}{|c|}{$\begin{array}{l}\text { Algunas } \\
\text { veces }\end{array}$} & \multicolumn{2}{|c|}{$\begin{array}{c}\text { Casi } \\
\text { siempre }\end{array}$} & \multicolumn{2}{|c|}{ Siempre } & \multicolumn{2}{|c|}{ Total } & \multirow{2}{*}{\multicolumn{2}{|c|}{$\overline{\mathrm{X}}$}} & \multirow{2}{*}{\multicolumn{2}{|c|}{$\sigma$}} \\
\hline & $\mathrm{Fa}$ & $\%$ & $\mathrm{Fa}$ & $\%$ & $\mathrm{Fa}$ & $\%$ & $\mathrm{Fa}$ & $\%$ & $\mathrm{Fa}$ & $\%$ & $\mathrm{Fa}$ & $\%$ & & & & \\
\hline & 7 & 16 & 6 & 14 & 8 & 18 & 12 & 27 & 11 & 25 & 44 & 100 & 3,27 & MEP & 1,41 & MAD \\
\hline $\begin{array}{l}\text { Gestión de } \\
\text { los recursos }\end{array}$ & 19 & 43 & 5 & 11 & 5 & 11 & 7 & 16 & 8 & 18 & 44 & 100 & 2,54 & PP & 1,59 & MAD \\
\hline $\begin{array}{l}\text { Generación } \\
\text { y selección } \\
\text { de ideas }\end{array}$ & 7 & 16 & 2 & 5 & 13 & 30 & 8 & 18 & 14 & 32 & 44 & 100 & 3,47 & $\mathrm{P}$ & 1,37 & ALD \\
\hline \multicolumn{13}{|c|}{ Media $(\bar{X})$ general de las prácticas de gestión } & \multicolumn{4}{|l|}{3,09} \\
\hline \multicolumn{13}{|c|}{ Categoría general de acuerdo con la Media $(\bar{X})$} & \multicolumn{4}{|c|}{$\begin{array}{l}\text { Medianamente presente } \\
(\mathrm{MEP})\end{array}$} \\
\hline \multicolumn{13}{|c|}{ Desviación estándar ( $\sigma$ ) general de prácticas de gestión } & \multirow{2}{*}{\multicolumn{4}{|c|}{1,51}} \\
\hline \multicolumn{13}{|c|}{ Categoría general de acuerdo con la Desviación estándar ( $\sigma$ ) } & \multicolumn{3}{|c|}{ Muy alta dispersión (MAD) } & \\
\hline
\end{tabular}

En primera instancia, la práctica de estrategia y cultura de innovación se evidencia que las respuestas de los informantes claves se ubican en las opciones casi siempre $(27 \%)$, siempre $(25 \%)$ y algunas veces $(18 \%)$, lo que indica una mediana presencia de esta práctica en las medianas empresas analizadas, con una media de 3,27 y una desviación estándar de 1,41, indicando una muy alta dispersión en las respuestas. En contraste, los estudios de González et al. (2014) y Gil et al. (2018) destacan que las prácticas más desarrolladas por las empresas son las asociadas con la cultura de innovación y la forma como esta se refleja en los lineamientos gerenciales. Así, la estrategia de innovación incluye un conjunto de acciones relacionadas con el desarrollo interno y la adquisición externa de conocimiento mediante las transacciones de mercado y la cooperación (Vega et al., 2009), por lo que la dirección estratégica de la innovación está determinada por las capacidades internas, los recursos y la base de conocimiento de la empresa (Norris \& Ciesielska, 2019). Recientemente, la literatura destaca que la innovación abierta requiere el desarrollo de redes internas y externas para gestionar el conocimiento disponible en el entorno de la organización (Álvarez y Bernal, 2017). Adicionalmente, la cultura de innovación permite explorar nuevos conocimientos para innovar (Naranjo y Calderon, 2018; Hanifah et al., 2019; Ghasemzadeh et al., 2019; Dabić et al., 2019; Hanifah et al., 2020). Del mismo modo, la cultura de innovación está correlacionada con los componentes del capital intelectual (humano, estructural y relacional) y es determinante para el éxito de la empresa (Dabić et al., 2019; Hanifah et al., 2020).

En segunda instancia, en la práctica de gestión de los recursos, la mayor parte de las respuestas se ubica en la opción nunca (43\%), lo que indica que este tipo de prácticas se encuentra poco presente en las medianas empresas analizadas, con una media de 2,54 y una desviación estándar de 1,59, denotando una muy alta dispersión en las respuestas. En contraste, González et al. (2014) establecen que la práctica más frecuente para gestionar recursos corresponde a la existencia de una unidad de innovación en la empresa, no obstante, la práctica menos observada corresponde a la disponibilidad de inversión en desarrollo experimental en I+D; lo que restringe el aprendizaje organizacional para innovar (González et al., 2014). En este sentido, los autores Díaz et al. (2014), Cainelli et al. (2015) y Da Costa et al. (2014) coinciden en la importancia de obtener recursos externos para innovar, mientras que, Díaz et al. (2014), González et al. (2014), Abdul y Medase (2019) y Fernandes et al. (2018) destacan la importancia de la comunicación interna y externa para asociar el conocimiento de las partes interesadas en el desarrollo de proyectos de innovación. En este contexto, es relevante la gestión de los recursos existentes de acuerdo con la estrategia de innovación y la mejora de las capacidades técnicas de la empresa (Meng y Brown, 2018).

En tercera instancia, en la práctica de generación y selección de ideas, el 32\% de las respuestas corresponden a la opción siempre y seguido del 30\% en la alternativa algunas veces, lo cual establece la presencia de esta práctica en las empresas analizadas, con una media de 3,47 y una desviación estándar de 1,37, denotando una alta dispersión. Estos resultados validan la importancia del fomento de la generación de ideas mediante la participación de los colaboradores en diferentes escenarios de innovación o a través de la implementación de filtros para priorizar las iniciativas en función de su factibilidad (González et al., 2014; Bäckström y Bengtsson, 2019). Igualmente, la literatura destaca la necesidad de establecer alianzas estratégicas, especialmente con la academia (González et al., 2014; Juliao et al., 2013). Recientemente, se destacan la efectividad de los procesos de ideación en conjunto con los clientes, mediante grupos focales o etnografía (Cooper, 2019); así como de las ideas derivadas de plataformas basadas en la web (Qian et al., 2019) y análisis de redes sociales, impulsando la innovación basada en datos (Bhimani et al., 2019) 
En suma, las prácticas de gestión de la innovación se encuentran medianamente presentes, con un promedio de 3,09 y una desviación estándar de 1,51, indicando una muy alta dispersión. Lo anterior, evidencia las deficiencias de las medianas empresas analizadas para implementar rutinas organizacionales que favorezcan la innovación, pese a la importancia de las misma para aumentar la efectividad en el proceso innovador y lograr la sostenibilidad en un entorno competitivo y globalizado.

\section{CONCLUSIONES}

De acuerdo al trabajo presentado y a los resultados obtenidos se pueden plantear las siguientes conclusiones. Primero, este articulo aporta evidencia relacionada con la importancia de la cultura organizacional, la formación y la claridad estratégica para favorecer el aprendizaje entre los miembros de la organización. En este sentido, se evidencia la participación activa de los colaboradores, directivos y miembros de las empresas en la solución de problemas, lo que fortalece la cultura de aprendizaje, necesaria para mejorar el desempeño. En este contexto, es relevante promover el desarrollo de nuevas habilidades en los equipos de trabajo, alineadas con los objetivos estratégicos de la empresa. Sin embargo, en las medianas empresas es necesario fortalecer la infraestructura de soporte a los procesos de aprendizaje organizacional, mediante la implementación de herramientas tecnológicas para la difusión y almacenamiento de los conocimientos adquiridos. Segundo, las medianas empresas analizadas implementan la generación y selección de ideas como práctica de gestión de innovación, sin embargo, presentan dificultades en la gestión de recursos para el desarrollo de proyectos innovadores, debido a la poca disponibilidad de recursos para invertir en procesos de investigación y desarrollo y a la baja utilización de mecanismos de propiedad intelectual para rentabilizar las innovaciones. Adicionalmente, las empresas deben fortalecer la interacción entre las áreas y sus redes externas para abordar temáticas relacionadas con la innovación.

\section{REFERENCIAS}

Abdul Basit, S., y Medase, K., The diversity of knowledge sources and its impact on firm-level innovation: Evidence from Germany, https://doi.org/10.1108/EJIM-10-2018-0232, European Journal of Innovation Management, 22(4), 681-714 (2019).

Álvarez-Aros, E. L., y Bernal-Torres, C. A., Modelo de innovación abierta: énfasis en el potencial humano, https://doi.org/10.4067/S0718-07642017000100007, Información Tecnológica, 28(1), 65-76 (2017).

Anlesinya, A., Organizational barriers to employee training and learning: evidence from the automotive sector, https://doi.org/10.1108/DLO-03-2017-0022, Development and Learning in Organizations, 32(3), 8-10 (2018).

Arasanmi, C. N., y Krishna, A., Employer branding: perceived organisational support and employee retention - the mediating role of organisational commitment, https://doi.org/10.1108/ICT-10-2018-0086, Industrial and Commercial Training, 51(3), 174-183 (2019).

Bäckström, I., y Bengtsson, L., A mapping study of employee innovation: proposing a research agenda, https://doi.org/10.1108/EJIM-05-2018-0101, European Journal of Innovation Management, 22(3), 468-492 (2019).

Berber, N., y Lekovic, B., The impact of HR development on innovative performances in central and eastern European countries, https://doi.org/10.1108/ER-08-2017-0188, Employee Relations, 40(5), 762-786 (2018).

Bhimani, H., Mention, A. L., y Barlatier, P. J., Social media and innovation: A systematic literature review and future research directions, https://doi.org/10.1016/j.techfore.2018.10.007, Technological Forecasting and Social Change, 144, 251-269 (2019).

Bravo-Ibarra, E. R., y Herrera, L., Capacidad de innovación y configuración de recursos organizativos, Intangible Capital, 301-320 (2009).

Cainelli, G., De Marchi, V., y Grandinetti, R., Does the development of environmental innovation require different resources? Evidence from Spanish manufacturing firms. Journal of Cleaner Production, 94, $211-220$ (2015).

Castañeda, D. I, Condiciones para el aprendizaje organizacional, Estudios Gerenciales, 31, 62-67 (2015).

Cavazotte, F., Moreno, V., y Turano, L.M., Cultura de aprendizagem contínua, atitudes e desempenho no trabalho: uma comparação entre empresas do setor público e privado. Rev. Adm. Pública, 49(6), 1555-1578 (2015).

Chen, Q., Magnusson, M., y Björk, J., Collective firm-internal online idea development: Exploring the impact of feedback timeliness and knowledge overlap, https://doi.org/10.1108/EJIM-02-2018-0045, European Journal of Innovation Management, 23(1), 13-39 (2019).

Chen, Qinglan, y Eriksson, T., The mediating role of decentralization between strategy and performance: Evidence from Danish firms, https://doi.org/10.1108/JOCM-05-2018-0128, Journal of Organizational Change Management, 32(4), 409425 (2019).

Chen, T., Hao, S., Ding, K., Feng, X., Li, G., y Liang, X., The impact of organizational support on employee performance, https://doi.org/10.1108/ER-01-2019-0079, Employee Relations, 42(1), 166-179 (2020). 
Clausen, T., Pohjola, M., Sappraserty, K., y Verspagen, B., Innovation strategies as a source of persistent innovation, Industrial and Corporate Change (2011).

Cooper, R. G., The drivers of success in new-product development, https://doi.org/10.1016/j.indmarman.2018.07.005, Industrial Marketing Management, 76, 36-47 (2019).

Córdoba, J. M., y Naranjo, J. C., Incidencia de la inversión en innovación en las ventas de productos innovadores. Evidencia empírica en empresas manufactureras de Colombia, https://doi.org/10.4067/S0718-07642017000200017, Informacion Tecnologica, 28(2), 153-166 (2017).

Da Costa, S., Páez, D., Sánchez, F., Gondim, S. y Rodríguez, M., Factores favorables a la innovación en las organizaciones: una integración de meta-análisis. Journal of Work and Organizational Psychology, 30, 67-74 (2014).

Dabić, M., Lažnjak, J., Smallbone, D., y Švarc, J., Intellectual capital, organisational climate, innovation culture, and SME performance: Evidence from Croatia, https://doi.org/10.1108/JSBED-04-2018-0117, Journal of Small Business and Enterprise Development, 26(4), 522-544 (2019).

Dias, T., de Souza, P. H., Moreira, M. F., y de Souza, W. V. B., The structure of an innovation ecosystem: foundations for future research, https://doi.org/10.1108/MD-03-2019-0383, Management Decision (2020).

Díaz, M., Martínez, H., Becerra, L., y Bravo, E., Rutinas de capacidad de innovación en los procesos financieros de las instituciones de educación superior - caso colombiano. Sotavento mba (23), 64-89 (2014).

Dobni, C. B., y Klassen, M., The decade of innovation: from benchmarking to execution, https://doi.org/10.1108/JBS-112019-0209, Journal of Business Strategy (2020).

Fernandes, M., Vasconcelos, S., y Dobelin, S., Literature on organizational innovation: past and future, https://doi.org/10.1108/inmr-01-2018-001, Innovation \& Management Review, 15(1), 2-19 (2018).

Ferreira, A. I., Cardoso, C., y Braun, T., The mediating effects of ego-resilience in the relationship between organizational support and resistance to change, https://doi.org/10.1108/BJM-06-2017-0171, Baltic Journal of Management, 13(1), 104-124 (2018).

Ghasemzadeh, P., Nazari, J. A., Farzaneh, M., y Mehralian, G., Moderating role of innovation culture in the relationship between organizational learning and innovation performance, https://doi.org/10.1108/TLO-08-2018-0139, The Learning Organization, 26(3), 289-303 (2019).

Gil, A. J., y Mataveli, M., The relevance of information transfer in learning culture: A multigroup study by firm size in the wine industry, https://doi.org/10.1108/MD-11-2016-0800, Management Decision, 55(8), 1698-1716 (2017).

Gil, A. J., Rodrigo-Moya, B., y Morcillo-Bellido, J., The effect of leadership in the development of innovation capacity: $A$ learning organization perspective, https://doi.org/10.1108/LODJ-12-2017-0399, Leadership and Organization Development Journal, 39(6), 694-711 (2018).

González, J., García, L., Lucero, C., y Romero, N., Estrategia y cultura de innovación, gestión de los recursos y generación de ideas: prácticas para gestionar la innovación en empresas, Pensamiento y gestión (36), 107-133 (2014).

Gupta, R. K., Kumar, C., y Sharma, R., Team building, employee empowerment and employee competencies: Moderating role of organizational learning culture, https://doi.org/10.1108/EJTD-08-2018-0086, European Journal of Training and Development, 43(1-2), 39-60 (2019).

Hanifah, H., Abdul Halim, H., Ahmad, N. H., y Vafaei-Zadeh, A., Emanating the key factors of innovation performance: leveraging on the innovation culture among SMEs in Malaysia, https://doi.org/10.1108/JABS-04-2018-0130, Journal of Asia Business Studies, 13(4), 559-587 (2019).

Hanifah, H., Halim, H. A., Ahmad, N. H., y Vafaei-Zadeh, A., Can internal factors improve innovation performance via innovation culture in SMEs?, https://doi.org/10.1108/BIJ-06-2018-0174, Benchmarking: An International Journal, 27(1), 382-405 (2020).

Hernandez, R., Fernandez, C., y Baptista, P., Metodología de la investigación, 6a Edicion, Mc Graw Hill, México. D.F (2014).

Ibrahim, R., Boerhannoeddin, A., y Bakare, K. K., The effect of soft skills and training methodology on employee performance, https://doi.org/10.1108/EJTD-08-2016-0066, European Journal of Training and Development, 41(4), 388406 (2017).

Juliao, J. L., Barrios Aguirre, F., Schmutzler, J. y Sánchez Manchola, I.D., Relación entre la estrategia de innovación de la firma y su decisión de patentar: evidencia de empresas pertenecientes al sector manufacturero colombiano, Estudios Gerenciales, 29, 313-321 (2013).

Liao, S., Fu, L., y Liu, Z., Investigating open innovation strategies and firm performance: the moderating role of technological capability and market information management capability, https://doi.org/10.1108/JBIM-01-2018-0051, Journal of Business and Industrial Marketing, 35(1), 23-39 (2020).

Malik, P., y Garg, P., The relationship between learning culture, inquiry and dialogue, knowledge sharing structure and affective commitment to change, https://doi.org/10.1108/JOCM-09-2016-0176, Journal of Organizational Change Management, 30(4), 610-631 (2017). 
Manresa, A., Bikfalvi, A., y Simon, A., The impact of training and development practices on innovation and financial performance, https://doi.org/10.1108/ICT-04-2019-0035, Industrial and Commercial Training, 51(7-8), 421-444 (2019).

Martínez Bencardino, C., Estadística y muestro, 13ª Ed., Ecoe Ediciones, Bogotá, Colombia (2012).

Meng, X., y Brown, A., Innovation in construction firms of different sizes: drivers and strategies, https://doi.org/10.1108/ECAM-04-2017-0067, Engineering, Construction and Architectural Management, 25(9), 12101225 (2018).

Naranjo-Valencia, J. C. y Calderón-Hernández, G., Construyendo una cultura de innovación. Una propuesta de transformación cultural, http://dx.doi.org/10.1016/j.estger.2014.12.005, Estudios Gerenciales, 31, 223-236 (2015).

Naranjo-Valencia, J. C., y Calderon-Hernández, G., Model of Culture for Innovation, https://doi.org/10.5772/intechopen.81002, In Organizational Culture, IntechOpen, 1st ed., 13-34 (2018).

Natalicchio, A., Messeni Petruzzelli, A., Cardinali, S., y Savino, T., Open innovation and the human resource dimension: An investigation into the Italian manufacturing sector, https://doi.org/10.1108/MD-03-2017-0268, Management Decision, 56(6), 1271-1284 (2018).

Norris, D., y Ciesielska, M., Towards a framework for innovation orientation within business and management studies: $A$ systematic review and paths for future research, https://doi.org/10.1108/JOCM-02-2018-0051, Journal of Organizational Change Management, 32(1), 123-144 (2019).

Overstreet, R. E., Skipper, J. B., Huscroft, J. R., Cherry, M. J., y Cooper, A. L., Multi-study analysis of learning culture, human capital and operational performance in supply chain management, https://doi.org/10.1108/jdal-11-2018-0017, Journal of Defense Analytics and Logistics, 3(1), 41-59 (2019).

Padachi, K., y Lukea Bhiwajee, S., Barriers to employee training in small and medium sized enterprises: Insights and evidences from Mauritius, https://doi.org/10.1108/EJTD-02-2014-0018, European Journal of Training and Development, 40(4), 232-247 (2016).

Roshchin, S., y Travkin, P., Determinants of on-the-job training in enterprises: the Russian case, https://doi.org/10.1108/EJTD-05-2017-0050, European Journal of Training and Development, 41(9), 758-775 (2017).

Singh, R., Organisational embeddedness as a moderator on the organisational support, trust and workplace deviance relationships, https://doi.org/10.1108/EBHRM-03-2019-0025, Evidence-Based HRM, 8(1), 1-17 (2019).

Stable-Rodríguez, Y, Aprendizaje organizacional en organizaciones de ciencia tecnología e innovación. Ingeniería Industrial, XXXVII (1), 78-90 (2016).

Suifan, T. S., Abdallah, A. B., y Al Janini, M., The impact of transformational leadership on employees' creativity: The mediating role of perceived organizational support, https://doi.org/10.1108/MRR-02-2017-0032, Management Research Review, 41(1), 113-132 (2018).

Tamayo, M., El proceso de la investigación científica, $5^{\mathrm{a}}$ Ed., Limusa S.A, México (2011).

Tang, G., Yu, B., Cooke, F. L., y Chen, Y., High-performance work system and employee creativity: The roles of perceived organisational support and devolved management, https://doi.org/10.1108/PR-09-2016-0235, Personnel Review, 46(7), 1318-1334 (2017).

Tarapuez, E., Guzmán, B.E., y Parra Hernández, R., Estrategia e innovación en las Mipymes colombianas ganadoras del premio Innova 2010-2013, Estudios Gerenciales, 32, 170-180 (2016).

Vega-Jurado, J., Gutiérrez-Gracia, A. y Fernández-de-Lucio, I., La relación entre las Estrategias de Innovación: Coexistencia o Complementariedad, Journal of technology management \& innovation, 4, 74-88 (2009).

Villaluz, V. C., y Hechanova, M. R. M., Ownership and leadership in building an innovation culture, https://doi.org/10.1108/LODJ-05-2018-0184, Leadership and Organization Development Journal, 40(2), 138-150 (2019).

Xiao, H., Shi, Y., y Varma, A., The effects of employee stock ownership plans on career development in a new era: Evidence from China's manufacturing transformation, https://doi.org/10.1108/CDI-05-2018-0126, Career Development International, 24(5), 453-474 (2019).

Xie, L., Dirani, K. M., Beyerlein, M., y Qiu, S., Learning culture in a Chinese SME: the unique role of work-family enrichment, https://doi.org/10.1108/EJTD-06-2019-0085, European Journal of Training and Development, 2046-9012 (2019).

Yap, J. B., y Toh, H., Investigating the principal factors impacting knowledge management implementation in construction organisations, https://doi.org/10.1108/JEDT-03-2019-0069, Journal of Engineering, Design and Technology, 18(1), 55-69 (2020). 